\title{
Invariant Subspaces, Dilation Theory, and the Structure of the Predual of a Dual Algebra. II
}

\author{
C. APOSTOL, H. BERCOVICI, C. FOIAS \\ \& C. PEARCY
}

1. Introduction. In the past few years operator theorists have been studying the structure of the preduals of various operator algebras, and thereby have made some remarkable advances in the areas of invariant subspaces, dilation theory, and reflexivity. (See the bibliography for a list of pertinent articles.) In this paper, which is a continuation of the sequence [12], [10], [6], we add to our knowledge of the structure of various preduals, and make some progress on the invariant subspace problem for contractions whose spectrum contains the unit circle. Most of the results herein were presented (by the fourth author) at the CBMS/NSF regional conference in Tempe, Arizona in May 1984.

The notation and terminology below agree with those in [12], [10], and [6]. For the benefit of the reader, we review a few pertinent definitions and earlier results. Let $\mathscr{H}$ be a separable, infinite dimensional, complex Hilbert space, and let $\mathscr{L}(\mathscr{H})$ denote the algebra of all bounded linear operators on $\mathscr{H}$. It is well known (cf. [21, p. 40]) that $\mathscr{L}(\mathscr{H})$ is the dual space of the ideal $\mathscr{C}_{1}(\mathscr{H})$ of trace-class operators under the bilinear functional

$$
\langle T, L\rangle=\operatorname{tr}(T L), \quad T \in \mathscr{L}(\mathscr{H}), L \in \mathscr{C}_{1}(\mathscr{H}) .
$$

A subalgebra $\mathscr{A}$ of $\mathscr{L}(\mathscr{H})$ that contains $1_{\mathscr{H}}$ and is closed in the weak* topology on $\mathscr{L}(\mathscr{H})$ is called a dual algebra. It follows from general principles that if $\mathscr{A}$ is a dual algebra, then $\mathscr{A}$ is the dual space of the quotient space $Q_{\mathscr{A}}=\mathscr{C}_{1}(\mathscr{H}) /{ }^{\perp} \mathscr{A}$ (where ${ }^{\perp} \mathscr{A}$ is the preannihilator in $\mathscr{C}_{1}(\mathscr{H})$ of $\mathscr{A}$ ) under the pairing

$$
\langle T,[L]\rangle=\operatorname{tr}(T L), \quad T \in \mathscr{A},[L] \in Q_{\mathscr{A}} .
$$

(Throughout the paper we write $[L]_{Q_{\mathscr{A}}}$, or simply $[L]$ when there is no possibility of confusion, for the image of a trace-class operator $L$ in the quotient space $Q_{\star}$.) If $x$ and $y$ are vectors in $\mathscr{H}$, then the associated rank-one operator $x \otimes y$ is defined by $(x \otimes y)(u)=(u, y) x, u \in \mathscr{H}$. Clearly $x \otimes y \in \mathscr{C}_{1}(\mathscr{H})$. Since every operator $L$ in $\mathscr{C}_{1}(\mathscr{H})$ can be written as $L=\sum_{i=1}^{\infty} x_{i} \otimes y_{i}$ for certain square summable se- 
quences $\left\{x_{i}\right\}_{i=1}^{\infty}$ and $\left\{y_{i}\right\}_{i=1}^{\infty}$ from $\mathscr{H}$ (with convergence in the trace norm, of course), it follows easily that every element of $Q_{\rtimes}$ has the form $\sum_{i=1}^{\infty}\left[x_{i} \otimes y_{i}\right]$. A dual algebra $\mathscr{A} \subset \mathscr{L}(\mathscr{H})$ is said to have property $\left(\mathbf{A}_{1}\right)$ if every element $[L]$ of $Q_{\mathscr{A}}$ can be written in the form $[L]=[x \otimes y]$ for certain vectors $x, y$ in $\mathscr{H}$. More generally, if $n$ is any nonzero cardinal number not exceeding $\aleph_{0}$, and if for every family $\left\{\left[L_{i j}\right]\right\}_{0 \leq i, j<n}$ of elements of $Q_{\mathscr{A}}$, one can find a pair of sequences $\left\{x_{i}\right\}_{0 \leq i<n}$ and $\left\{y_{i}\right\}_{0 \leq j<n}$ of vectors from $\mathcal{H}$ such that

$$
\left[L_{i j}\right]=\left[x_{i} \otimes y_{i}\right] \quad 0 \leq i, j<n,
$$

then $\mathscr{A}$ is said to have property $\left(\mathbf{A}_{n}\right)$. If $\mathbf{T}=\{\zeta \in \mathbf{C}:|\zeta|=1\}, T$ is an absolutely continuous contraction in $\mathscr{L}(\mathscr{H})$ (i.e., a contraction whose unitary direct summand is either absolutely continuous or acts on the space (0)), and $\mathscr{A}_{T}$ denotes the dual algebra generated by $T$, then, by definition (cf. [12]), $T$ belongs to the class $\mathbf{A}=\mathbf{A}(\mathscr{H})$ if the Sz.-Nagy-Foias $H^{\infty}(\mathbf{T})$-functional calculus $\Phi_{T}$ is an isometry of $H^{\infty}(\mathbf{T})$ onto $\mathscr{A}_{T}$. In this case, one knows (cf. [19]) that there exists an isometry $\phi_{T}$ of $Q_{T}=Q_{A_{T}}$ onto $L^{1}(\mathbf{T}) / H_{0}^{1}(\mathbf{T})$ (the predual of $H^{\infty}(\mathbf{T})$ ) such that $\phi_{T}^{*}=\Phi_{T}$. Moreover if $T \in \mathbf{A}$, the pair of spaces $\left\{\mathbb{A}_{T}, Q_{T}\right\}$ can be identified with the pair $\left\{H^{\infty}(\mathbf{T}), L^{1}(\mathbf{T}) / H_{0}^{1}(\mathbf{T})\right\}$ via the pair of isometries $\left\{\Phi_{T}, \phi_{T}\right\}$. If $T \in \mathbf{A}$ and $\mathscr{A}_{T}$ has property $\left(\mathbf{A}_{n}\right)$ for some $1 \leq n \leq \aleph_{0}$, we say that $T$ belongs to the class $\mathbf{A}_{n}$. In [12] a very useful dilation theory was developed for the operators $T$ belonging to some class $\mathbf{A}_{n}$, and in [34] and [13] it was shown that the (BCP)-operators (first studied in [19]) belong to the class $\mathbf{A}_{\aleph_{0}}$. Furthermore, in [6] it was shown that $C_{00} \cap \mathbf{A}_{1}=C_{00} \cap \mathbf{A}_{\aleph_{0}}$ (where $C_{00}$ is the set of all contractions $T$ in $\mathscr{L}(\mathscr{H})$ such that both sequences $\left\{T^{n}\right\}$ and $\left\{T^{* n}\right\}$ converge to zero in the strong operator topology), and several sufficient conditions of a spectral and topological nature were given in order that an operator belong to $\mathbf{A}_{\aleph_{0}}$.

The purpose of this paper is to improve some of the sufficient conditions for membership in $\mathbf{A}_{\aleph_{0}}$ that we obtained in [6], with the aim of making progress toward the verification of our conjecture ([6, Conjecture 2.14]) that $\mathbf{A}=\mathbf{A}_{1}$. As was seen in [6, Proposition 2.15], a consequence of the validity of the relation $\mathbf{A}=\mathbf{A}_{1}$ would be that every contraction $T$ in $\mathscr{L}(\mathscr{H})$ whose spectrum $\sigma(T)$ contains the unit circle $\mathbf{T}$ has a nontrivial invariant subspace, and this provides a major motivation for us to continue to try to establish this relation.

2. Weak density and membership in $\boldsymbol{A}_{\aleph_{0}}$. If the relation $\mathbf{A}=\mathbf{A}_{1}$ is true, then, of course, $\mathbf{A} \cap C_{00}=\mathbf{A}_{\aleph_{0}} \cap C_{00}$ by the result mentioned above from [6]. Thus it makes sense to try to establish the relation $\mathbf{A} \cap C_{00} \subset \mathbf{A}_{\aleph_{0}}$ and we concentrate on this problem. If $T \in \mathscr{L}(\mathscr{H})$, then by the weak topology on $Q_{T}$ we mean, of course, the topology induced on $Q_{T}$ by its dual space $\mathscr{A}_{T}$. The following result improves [6, Lemma. 2.8].

Theorem 2.1. Suppose $T \in \mathbf{A} \cap C_{00}$ and the set

$$
\Lambda=\left\{[x \otimes y] \in Q_{T}:\|x\|,\|y\| \leq 1\right\}
$$

is weakly sequentially dense in some ball of positive radius about the origin in $Q_{T}$. Then $T \in \mathbf{A}_{\aleph_{0}}$. 
Proof. Suppose that every element in the open ball $B_{0, \delta}$ of radius $\delta>0$ centered at the origin in $Q_{T}$ is a weak limit of a sequence from the set $\Lambda$ in (4), and let $\varepsilon$ be any positive number chosen sufficiently small that $20 \varepsilon<\min \{\delta, 1\}$. Since $T \oplus T \in C_{00}(\mathscr{H} \oplus \mathscr{H})$, by [6, Theorem 2.4] it suffices to show that $T \oplus T \in$ $\mathbf{A}_{\aleph_{0}}(\mathscr{H} \oplus \mathscr{H})$, and by [6, Lemma 2.8], to accomplish this it suffices to show that if $[\tilde{L}] \in Q_{T \oplus T}$ and satisfies $\|[\tilde{L}]\|<\delta / 20$, then there exist vectors $\tilde{x}$ and $\tilde{y}$ in the unit ball of $\mathscr{H} \oplus \mathscr{H}$ such that

$$
\|[\tilde{L}]-[\tilde{x} \otimes \tilde{y}]\|_{Q_{T \oplus T}}<\varepsilon .
$$

Using the existence of the isometries $\Phi: \mathscr{A}_{T} \rightarrow \mathscr{A}_{T \oplus T}$ and $\phi: Q_{T \oplus T} \rightarrow Q_{T}$ such that $\phi^{*}=\Phi[6$, Theorem 1.10], and formula (44) from [6], we see that to establish (5), it suffices to show that if $[L] \in Q_{T}$ and satisfies $\|[L]\|<\delta$, then there exist vectors $x, y, s, t$ in $\mathscr{H}$ satisfying

$$
\|x\|,\|y\| \leq 1, \quad\|s\|,\|t\| \leq \sqrt{5}
$$

and

$$
\|[L]-[x \otimes y]-[s \otimes t]\|<20 \varepsilon .
$$

(For, in this case, given $[\tilde{L}]$ in $Q_{T \oplus T}$ with $\|[\tilde{L}]\|<\delta / 20$, we set $[L]=20 \phi([\tilde{L}])$, find vectors $x, y, s, t$ satisfying (6) and (7), define

$$
\tilde{x}=(x / 2 \sqrt{5}) \oplus(s / 2 \sqrt{5}), \quad \tilde{y}=(y / 2 \sqrt{5}) \oplus(t / 2 \sqrt{5}),
$$

and use (44) of [6] to obtain (5).) To find appropriate vectors $x, y, s, t$ in $\mathscr{H}$ satisfying (6) and (7), we choose a sequence $\left\{\left[x_{n} \otimes y_{n}\right]\right\}$ from $\Lambda$ that converges weakly to $[L]$. Without loss of generality we may suppose that the sequences $\left\{x_{n}\right\}$ and $\left\{y_{n}\right\}$ are weakly convergent in $\mathscr{H}_{\text {- }}$-say to $x$ and $y$, respectively, and we note that $\|x\|,\|y\| \leq 1$. For $n \in \mathbf{N}$ we define $s_{n}=x_{n}-x$ and $t_{n}=y_{n}-y$. The sequences $\left\{s_{n}\right\}$ and $\left\{t_{n}\right\}$ converge weakly to zero in $\mathscr{H}$, and since

$$
\left[s_{n} \otimes t_{n}\right]=\left[x_{n} \otimes y_{n}\right]-\left[s_{n} \otimes y\right]-\left[x \otimes t_{n}\right]-[x \otimes y]
$$

and $\left\|\left[s_{n} \otimes y\right]\right\|+\left\|\left[x \otimes t_{n}\right]\right\| \rightarrow 0$ by [6, Proposition 2.1$]$, the sequence $\left\{\left[s_{n} \otimes t_{n}\right]\right\}$ converges weakly to $[L]-[x \otimes y]$. Using [6, Proposition 2.1] once again, we may choose subsequences $\left\{s_{n(k)}\right\}_{k=1}^{\infty}$ and $\left\{t_{n(k)}\right\}_{k=1}^{\infty}$ of $\left\{s_{n}\right\}$ and $\left\{t_{n}\right\}$, respectively, such that

$$
\left\|\left[s_{n(j)} \otimes t_{n(k)}\right]\right\|,\left\|\left[s_{n(j)} \otimes s_{n(k)}\right]\right\|,\left\|\left[t_{n(j)} \otimes t_{n(k)}\right]\right\|<\varepsilon / 2^{j+k}, \quad j, k \in \mathbf{N}, j \neq k .
$$

(First one chooses $n(1)=1$, then chooses $n(2)$ so $s_{n(2)}$ and $t_{n(2)}$ satisfy the appropriate six relations to $s_{n(1)}$ and $t_{n(1)}$, etc.) Since $[L]-[x \otimes y]$ belongs to the weak closure of the set $\left\{\left[s_{n(k)} \otimes t_{n(k)}\right]: k \in \mathbf{N}\right\}$, it follows (cf. [16, Prob. 15L]) that $[L]-[x \otimes y]$ belongs to the norm closure of the convex hull of $\left\{\left[s_{n(k)} \otimes t_{n(k)}\right]: k \in \mathbf{N}\right\}$, and hence there exist non-negative numbers $c_{1}, \ldots, c_{m}$ such that $\sum_{j=1}^{m} c_{j}=1$ and

$$
\left\|[L]-[x \otimes y]-\sum_{j=1}^{m} c_{j}\left[s_{n(j)} \otimes t_{n(j)}\right]\right\|<\varepsilon .
$$


We define $s=\sum_{j=1}^{m} \sqrt{c_{j}} s_{n(j)}, t=\sum_{j=1}^{m} \sqrt{c_{j}} t_{n(j)}$, and compute, using (8):

$$
\begin{aligned}
&\|[L]-[x \otimes y]-[s \otimes t]\| \\
& \leq\left\|[L]-[x \otimes y]-\sum_{j=1}^{m} c_{j}\left[s_{n(j)} \otimes t_{n(j)}\right]\right\|+\left\|\sum_{\substack{i, j=1 \\
i \neq j}}^{m} \sqrt{c_{i}} \sqrt{c_{j}}\left[s_{n(i)} \otimes t_{n(j)}\right]\right\| \\
& \leq \varepsilon+\sum_{\substack{i, j=1 \\
i \neq j}}^{m}\left\|\left[s_{n(i)} \otimes t_{n(j)}\right]\right\| \leq 2 \varepsilon \leq 20 \varepsilon
\end{aligned}
$$

and

$$
\begin{aligned}
\|s\|^{2} & =\sum_{j=1}^{m} c_{j}\left\|s_{n(j)}\right\|^{2}+\sum_{\substack{i, j=1 \\
i \neq j}}^{m} \sqrt{c_{i}} \sqrt{c_{j}}\left(s_{n(i)}, s_{n(j)}\right) \\
& \leq 4+\sum_{\substack{i, j=1 \\
i \neq j}}^{m}\left\|\left[s_{n(i)} \otimes s_{n(j)}\right]\right\| \leq 4+\varepsilon \leq 5 .
\end{aligned}
$$

Since $\|t\| \leq \sqrt{5}$ by a similar computation, we have shown that (7) holds with vectors $x, y, s, t$ that satisfy (6), so the proof is complete.

Problem 2.2. Is the hypothesis of Theorem 2.1 valid for every $T$ in $\mathbf{A} \cap C_{00}$ ? We show shortly that for all such $T$ the set $\Lambda$ is always weakly dense in the unit ball of $Q_{T}$, so if that weak density could be improved to weak sequential density, it would prove that $\mathbf{A} \cap C_{00}=\mathbf{A}_{\aleph_{0}} \cap C_{00}$.

If $A \in \mathscr{L}(\mathscr{H})$ we write $|\sigma(A)|$ and $\left|\sigma_{e}(A)\right|$ for the spectral radius and essential spectral radius, respectively, of $A$, and we write $\|A\|_{e}$ for the essential norm of $A$, i.e., for the norm of $\pi(A)$ in the Calkin algebra.

The next proposition is a useful tool in many situations.

Proposition 2.3. If $T \in \mathbf{A}$, then for all functions $f$ in $H^{\infty}(\mathbf{T})$,

$$
\left|\sigma_{e}(f(T))\right|=|\sigma(f(T))|=\|f(T)\|_{e}=\|f(T)\|=\|f\|_{\infty} .
$$

Proof. Since $T \in$ A we have $\|f(T)\|=\|f\|_{\infty}$ for all $f$ in $H^{\infty}(\mathbf{T})$, and by virtue of the well-known inequalities between the other quantities in (9), it obviously suffices to prove that $\left|\sigma_{e}(f(T))\right|=\|f\|_{\infty}$ for all $f$ in $H^{\infty}(\mathrm{T})$. The following chain of equalities shows that $|\sigma(f(T))|=\|f\|_{\infty}$ for all such $f$ :

$$
|\sigma(f(T))|=\lim _{n}\left\|f(T)^{n}\right\|^{1 / n}=\lim _{n}\left\|f^{n}(T)\right\|^{1 / n}=\lim _{n}\left\|f^{n}\right\|_{\infty}^{1 / n}=\|f\|_{\infty} .
$$

To show that $\left|\sigma_{e}(f(T))\right|=|\sigma(f(T))|$ for all $f$ in $H^{\infty}(\mathbf{T})$, and thereby complete the proof, we suppose, to the contrary, that there exists some $f_{0}$ in $H^{\infty}(\mathbf{T})$ such that $\left|\sigma_{e}\left(f_{0}(T)\right)\right|<\left|\sigma\left(f_{0}(T)\right)\right|$.

Let $\mu \in \sigma\left(f_{0}(T)\right)$ and satisfy 


$$
|\mu|=\left|\sigma\left(f_{0}(T)\right)\right|=\left\|f_{0}\right\|_{\infty} .
$$

Then clearly $\mu$ must be in the unbounded component of $\mathbf{C} \backslash \sigma_{e}\left(f_{0}(T)\right)$, so $\mu$ is an eigenvalue of $f_{0}(T)$ and the eigenspace $\mathscr{E}_{\mu}$ associated with $\mu$ is finite dimensional (cf. [33, Prop. 1.27]). Furthermore, $\mathscr{E}_{\mu}$ is an invariant (even hyperinvariant) subspace for $T$, and clearly we have

$$
\hat{f}_{0}\left(T \mid \mathscr{E}_{\mu}\right)=f_{0}\left(T \mid \mathscr{E}_{\mu}\right)=f_{0}(T) \mid \mathscr{E}_{\mu}=\mu \cdot 1_{\mathscr{E}_{\mu}},
$$

where $\hat{f}_{0}$ is the analytic extension of $f_{0}$ to the open unit disc D. Thus, by the spectral mapping theorem for operators on a finite-dimensional space, there must be an eigenvalue $\lambda_{0}$ of $T \mid \mathscr{E}_{\mu}$ such that $\hat{f}_{0}\left(\lambda_{0}\right)=\mu$, and since $T$ is an absolutely continuous contraction, $\left|\lambda_{0}\right|<1$. Thus $\lambda_{0} \in \mathbf{D}$, and since $\hat{f}_{0}$ is an open mapping on $\mathbf{D}, \hat{f}_{0}(\mathbf{D})$ must contain $\mu$ as an interior point. This contradicts (10), and thus the proof is complete.

The following result is the first step in our proof of weak density.

Proposition 2.4. Suppose $\mathcal{M}$ is a weak* closed linear manifold in $\mathscr{L}(\mathscr{H})$, $Q_{\mathcal{M}}=\mathscr{C}_{1}(\mathscr{H}) /{ }^{\perp} \mathcal{M}$ is its predual, and there exist sequences $\left\{x_{n}\right\}$ and $\left\{y_{n}\right\}$ in the unit ball of $\mathscr{H}$ such that

$$
\sup _{n \geq N}\left|\left(A x_{n}, y_{n}\right)\right|=\|A\|, \quad A \in \mathcal{M}, N \in \mathbf{N},
$$

and

$$
\begin{gathered}
\left|\left(x_{m}, x_{n}\right)\right|,\left|\left(y_{m}, y_{n}\right)\right| \leq 1 / 2^{m+n}, \quad m, n \in \mathbf{N}, m \neq n, \\
\left\|\left[x_{m} \otimes x_{n}\right]\right\|_{Q_{\mu}},\left\|\left[y_{m} \otimes y_{n}\right]\right\|_{Q_{\mu}},\left\|\left[x_{m} \otimes y_{n}\right]\right\|_{Q_{\mu}} \leq 1 / 2^{m+n}, \quad m, n \in \mathbf{N}, m \neq n .
\end{gathered}
$$

Then, for every $[L] \in Q_{\mu}$ and every $\varepsilon>0$, there exist vectors $u$ and $v$ in $\mathscr{H}$ such that $[u \otimes v]=[L]$ and $\|u\|,\|v\| \leq(1+\varepsilon)\|[L]\|^{1 / 2}$.

Proof. It clearly suffices to assume that $\|[L]\|=1$ and to find vectors $u$ and $v$ such that $[u \otimes v]=[L]$ and $\|u\|,\|v\| \leq 1+\varepsilon$. Choose $\delta$ in the interval $(0,1)$ so small that

$$
(1+\delta)^{1 / 2}\left(1+\delta^{1 / 2}+\delta+\delta^{3 / 2}+\ldots\right)<1+\varepsilon .
$$

We will choose, by induction, sequences $\left\{u_{n}\right\}_{n=0}^{\infty}$ and $\left\{v_{n}\right\}_{n=0}^{\infty}$ in $\mathscr{H}$ such that

$$
\begin{gathered}
\left\|[L]-\left[u_{n} \otimes v_{n}\right]\right\| \leq \delta^{n}, \quad n=0,1,2, \ldots, \\
\left\|u_{n}\right\|,\left\|v_{n}\right\| \leq(1+\delta)^{1 / 2}\left(1+\delta^{1 / 2}+\ldots+\delta^{(n-1) / 2}\right), \quad n=1,2, \ldots,
\end{gathered}
$$

and

$$
\left\|u_{n}-u_{n-1}\right\|,\left\|v_{n}-v_{n-1}\right\| \leq \delta^{(n-1) / 2}(1+\delta)^{1 / 2}, \quad n=1,2, \ldots .
$$

Suppose, for the moment, that these sequences $\left\{u_{n}\right\}$ and $\left\{v_{n}\right\}$ have been constructed to satisfy the above inequalities. It is obvious from (16) that the sequences $\left\{u_{n}\right\}$ and $\left\{v_{n}\right\}$ are Cauchy, and therefore converge-say to $u$ and $v$. Then $[L]=$ $[u \otimes v]$ from (14), and $\|u\|,\|v\| \leq 1+\varepsilon$ from (15) and (13). Thus, to complete 
the proof, it suffices to construct, by induction, the sequences $\left\{u_{n}\right\}$ and $\left\{v_{n}\right\}$ satisfying (14), (15), and (16). We set $u_{0}=v_{0}=0$, and suppose that $\left\{u_{0}, u_{1}, \ldots, u_{k}\right\}$ and $\left\{v_{0}, v_{1}, \ldots, v_{k}\right\}$ have been constructed to satisfy (14), (15), (16) for the appropriate values of $n$, and the further induction hypothesis that each $u_{i}, 0 \leq i \leq k$, is some finite linear combination of vectors in the sequence $\left\{x_{n}\right\}_{n=1}^{\infty}$ with coefficients at most one in modulus, and similarly for the $v_{i}$ relative to the sequence $\left\{y_{n}\right\}_{n=1}^{\infty}$. If $[L]=\left[u_{k} \otimes v_{k}\right]$, we set $u_{k+1}=v_{k+1}=0$ and the induction and the proof are complete. Otherwise, write $u_{k}=\sum_{i=1}^{M} \alpha_{i} x_{i}$ and $v_{k}=\sum_{i=1}^{M} \beta_{i} y_{i}$ where $\left|\alpha_{i}\right|$, $\left|\beta_{i}\right| \leq 1$ for $1 \leq i \leq M$, and choose an integer $N \geq M$ so large that

$$
\sum_{i \geq 1, j \geq N} 1 / 2^{i+j} \leq \delta^{k+1} / 4
$$

note that this implies that

$$
\sum_{i \geq N, j \geq 1} 1 / 2^{i+j} \leq \delta^{k+1} / 4 \text { and } \sum_{i, j=n}^{\infty} 1 / 2^{i+j}<\delta^{k+1} / 4 .
$$

Observe that

$$
\left\|\left([L]-\left[u_{k} \otimes v_{k}\right]\right) / \delta^{k}\right\| \leq 1,
$$

and note from (11) and [6, Proposition 2.1] that the closed absolutely convex hull $\overline{\operatorname{aco}}\left\{\left[x_{n} \otimes y_{n}\right]\right\}_{n \geq N+1}$ of the set $\left\{\left[x_{n} \otimes y_{n}\right]\right\}_{n \geq N+1}$ is the closed unit ball in $Q_{\mu}$. Hence there exist complex numbers $\gamma_{1}, \ldots, \gamma_{m}$ such that $\sum_{i=1}^{m}\left|\gamma_{i}\right| \leq 1$ and

$$
\left\|\left\{\left([L]-\left[u_{k} \otimes v_{k}\right]\right) / \delta^{k}\right\}-\sum_{i=1}^{m} \gamma_{i}\left[x_{N+i} \otimes y_{N+i}\right]\right\|<\delta / 4 .
$$

Hence

$$
\left\|[L]-\left[u_{k} \otimes v_{k}\right]-\sum_{i=1}^{m} \delta^{k} \gamma_{i}\left[x_{N+i} \otimes y_{N+i}\right]\right\|<\delta^{k+1} / 4 .
$$

Choose $\mu_{i}$ so that $\mu_{i}^{2}=\gamma_{i}, 1 \leq i \leq m$, and define

$$
u_{k+1}=u_{k}+\sum_{i=1}^{m} \delta^{k / 2} \mu_{i} x_{N+i}, \quad v_{k+1}=v_{k}+\sum_{i=1}^{m} \delta^{k / 2} \bar{\mu}_{i} y_{N+i} .
$$

By the way $N$ and the $\mu_{i}$ were defined it is clear that $u_{k+1}$ and $v_{k+1}$ are finite linear combinations of the $\left\{x_{n}\right\}$ and $\left\{y_{n}\right\}$ with coefficients in modulus at most one. Furthermore,

$$
\begin{aligned}
\left\|u_{k+1}-u_{k}\right\|^{2} & =\delta^{k}\left\|\sum_{i=1}^{m} \mu_{i} x_{N+i}\right\|^{2} \\
& \leq \delta^{k}\left(\sum_{i=1}^{m}\left|\mu_{i}\right|^{2}\left\|x_{N+i}\right\|^{2}+\sum_{\substack{i, j=1 \\
i \neq j}}^{m}\left|\mu_{i} \mu_{j}\left(x_{N+i}, x_{N+j}\right)\right|\right)
\end{aligned}
$$




$$
\leq \delta^{k}\left(1+\sum_{i, j=N}^{\infty} 1 / 2^{i+j}\right) \leq \delta^{k}\left(1+\frac{\delta^{k+1}}{4}\right) \leq \delta^{k}(1+\delta),
$$

and similarly for $\left\|v_{k+1}-v_{k}\right\|$, so (16) is satisfied for $n=k+1$, and since (15) is satisfied for $n=k$ by the induction hypothesis, the inequality

$$
\left\|u_{k+1}\right\| \leq\left\|u_{k+1}-u_{k}\right\|+\left\|u_{k}\right\|,
$$

together with the corresponding inequality for $\left\|v_{k+1}\right\|$, shows that (15) is valid for $n=k+1$. It remains only to show that (14) is valid for $n=k+1$, and we compute, using (17):

$$
\begin{aligned}
\left\|[L]-\left[u_{k+1} \otimes v_{k+1}\right]\right\| \leq & \left\|[L]-\left[u_{k} \otimes v_{k}\right]-\sum_{i=1}^{m} \delta^{k} \gamma_{i}\left[x_{N+i} \otimes y_{N+i}\right]\right\| \\
& +\left\|\left[u_{k} \otimes\left(\sum_{i=1}^{m} \delta^{k / 2} \bar{\mu}_{i} y_{N+i}\right)\right]\right\|+\left\|\left(\sum_{i=1}^{m} \delta^{k / 2} \mu_{i} x_{N+i}\right) \otimes v_{k}\right\| \\
& +\left\|\sum_{\substack{i, j=1 \\
i \neq j}}^{m} \delta^{k} \mu_{i} \mu_{j}\left[x_{N+i} \otimes y_{N+j}\right]\right\| \\
\leq & \delta^{k+1} / 4+\sum_{\substack{i \geq 1 \\
j \geq N}} 1 / 2^{i+j}+\sum_{\substack{i \geq N \\
j \geq 1}} 1 / 2^{i+j}+\sum_{i, j=N}^{\infty} 1 / 2^{i+j} \\
\leq & 4\left(\delta^{k+1} / 4\right)=\delta^{k+1},
\end{aligned}
$$

where we have made very generous estimates. Thus (14) is valid for $n=k+1$ and the induction may be continued. This completes the proof.

The following result is an immediate consequence of Proposition 2.4 and the fact that $\mathbf{A}_{1} \cap C_{00}=\mathbf{A}_{\aleph_{0}} \cap C_{00}$. We omit the proof.

Corollary 2.5. Suppose $T \in \mathbf{A}(\mathcal{H}) \cap C_{00}$ and there exist sequences $\left\{x_{n}\right\}$ and $\left\{y_{n}\right\}$ in the unit ball of $\mathscr{H}$ such that

$$
\sup _{n \geq N}\left|\left(f(T) x_{n}, y_{n}\right)\right|=\|f\|_{\infty}, \quad f \in H^{\infty}(\mathbf{T}), N \in \mathbf{N},
$$

and

(19) $\left\|\left[x_{m} \otimes x_{n}\right]\right\|,\left\|\left[y_{m} \otimes y_{n}\right]\right\|,\left\|\left[x_{m} \otimes y_{n}\right]\right\|<1 / 2^{m+n}, \quad m, n \in \mathbf{N}, m \neq n$.

Then $T \in \mathbf{A}_{\aleph_{0}}$.

The following result is another nice corollary of Proposition 2.4.

Proposition 2.6. Suppose $\mathcal{M}$ is a finite-dimensional subspace of $\mathscr{L}(\mathscr{H})$ and each $A$ in $\mathcal{M}$ satisfies $\|A\|=\|A\|_{e}$. Then, for every $\varepsilon>0$ and every $[L] \in Q_{\mathcal{M}}$, there exist $u$ and $v$ in $\mathscr{H}$ such that $\|u\|,\|v\| \leq(1+\varepsilon)\|[L]\|^{1 / 2}$ and

$$
[u \otimes v]_{Q_{\mu}}=[L]_{Q_{\mu}} \text {. }
$$


Proof. By Proposition 2.4, it suffices to construct sequences $\left\{x_{n}\right\}_{n=1}^{\infty}$ and $\left\{y_{n}\right\}_{n=1}^{\infty}$ in the unit ball of $\mathscr{H}$ such that (11) and (12) are satisfied. To this end, let $\left\{B_{j}\right\}_{j=1}^{\infty}$ be a countable set of nonzero operators norm dense in the unit ball of $\mathcal{M}$. Then, of course, it suffices to choose the sequences $\left\{x_{n}\right\}$ and $\left\{y_{n}\right\}$ to satisfy (12) and the condition

$$
\sup _{n \geq N}\left|\left(B_{j} x_{n}, y_{n}\right)\right|=\left\|B_{j}\right\|, \quad j \in \mathbf{N}, N \in \mathbf{N} .
$$

For each fixed $j \in \mathbf{N}$, one sees easily, using the equality $\left\|B_{j}\right\|=\left\|B_{j}\right\|_{e}$, that there exists an orthonormal sequence $\left\{e_{i}^{(j)}\right\}_{i=1}^{\infty}$ satisfying $\left\|B_{j} e_{i}^{(j)}\right\| \neq 0$ and $\left\|B_{j} e_{i}^{(j)}\right\| \rightarrow$ $\left\|B_{j}\right\| \neq 0$. Define $f_{i}^{(j)}=B_{j} e_{i}^{(j)} /\left\|B_{j} e_{i}^{(j)}\right\|$, and note that the sequence $\left\{f_{i}^{(j)}\right\}_{i=1}^{\infty}$ converges weakly to zero in $\mathscr{H}$ and satisfies

$$
\left(B_{j} e_{i}^{(j)}, f_{i}^{(j)}\right) \rightarrow\left\|B_{j}\right\| .
$$

We will choose the sequence $\left\{x_{n}\right\}$ from the set $\left\{e_{i}^{(j)}\right\}_{i, j}$, and, having chosen a given $x_{n}$, we will choose $y_{n}$ to be the corresponding $f_{i}^{(j)}$. For this purpose, we form the rectangular array:

$$
\begin{aligned}
& e_{1}^{(1)}, e_{2}^{(1)}, e_{3}^{(1)}, \ldots \\
& e_{1}^{(2)}, e_{2}^{(2)}, e_{3}^{(2)}, \ldots \\
& e_{1}^{(3)}, e_{2}^{(3)}, e_{3}^{(3)}, \ldots
\end{aligned}
$$

In view of (21), to ensure that (20) is satisfied it suffices to choose the sequence $\left\{x_{n}\right\}$ so that infinitely many terms from each row in (22) occur as terms of $\left\{x_{n}\right\}$. Thus, we will choose $x_{1}$ and $x_{2}$ to be different terms from the first row, $x_{3}$ from the second row, $x_{4}$ another term from the first row, $x_{5}$ another term from the second row, $x_{6}$ from the third row, etc. Thus our problem is to choose the $x_{n}$ (and the corresponding $y_{n}=f_{i}^{(j)}$ ) in this fashion so that (12) is also satisfied. Observe now that if $z \in \mathscr{H}$ and $\left\{w_{n}\right\}$ converges weakly to zero in $\mathscr{H}$, then $\left\{\left[z \otimes w_{n}\right]\right\}$ and $\left\{\left[w_{n} \otimes z\right]\right\}$ converge weakly to zero in $Q_{\mu}$. (For, if $A$ is fixed in $\mathcal{M}$, then $\left\langle A,\left[z \otimes w_{n}\right]\right\rangle=\left(A z, w_{n}\right) \rightarrow 0$.) Moreover, since $\operatorname{dim} Q_{\mu}=\operatorname{dim} M$ is finite, the weak and norm topologies coincide on $Q_{\mu}$, so also

$$
\left\|\left[z \otimes w_{n}\right]\right\|+\left\|\left[w_{n} \otimes z\right]\right\| \rightarrow 0 .
$$

Thus, since all rows in (22), and all rows in the corresponding array $\left\{f_{i}^{(j)}\right\}_{i, j}$, converge weakly to zero, it is easy to choose the $x_{n}$ (and $y_{n}$ ) by induction to satisfy (12), since at each step of the induction process there are only a finite number of inequalities to be satisfied. This completes the proof.

Corollary 2.7. Suppose $T \in \mathbf{A}, f_{1}, \ldots, f_{n} \in H^{\infty}(\mathbf{T})$, and $\varepsilon$ is an arbitrary positive number. Then, for each $[L] \in Q_{T}$, there exists vectors $u$ and $v$ in $\mathscr{H}$ such that $\|u\|,\|v\| \leq(1+\varepsilon)\|[L]\|_{Q_{T}}^{1 / 2}$ and

$$
\left\langle f_{i}(T),[u \otimes v]_{Q_{T}}\right\rangle=\left\langle f_{i}(T),[L]_{Q_{T}}\right\rangle, \quad i=1, \ldots, n .
$$


Proof. Let $M \subset \mathscr{A}_{T}$ be the finite-dimensional subspace of $\mathscr{L}(\mathscr{H})$ spanned by $f_{1}(T), \ldots, f_{n}(T)$. By Proposition 2.3 , the hypotheses of Proposition 2.6 are satisfied, so if $L \in \mathscr{C}_{1}(\mathscr{H})$, then there exist vectors $u$ and $v$ in $\mathscr{H}$ such that $\|u\|$, $\|v\| \leq(1+\varepsilon)\|[L]\|_{Q_{\mu}}^{1 / 2}$ and $[u \otimes v]_{Q_{\mu}}=[L]_{Q_{\mu}}$. Thus for $i=1,2, \ldots, n$,

$$
\begin{aligned}
\left\langle f_{i}(T),[u \otimes v]_{Q_{T}}\right\rangle & =\left(f_{i}(T) u, v\right)=\left\langle f_{i}(T),[u \otimes v]_{Q_{M}}\right\rangle \\
& =\left\langle f_{i}(T),[L]_{Q_{\mu}}\right\rangle=\operatorname{tr}\left(f_{i}(T) L\right)=\left\langle f_{i}(T),[L]_{Q_{T}}\right\rangle,
\end{aligned}
$$

so (23) is satisfied. Moreover, since for any $L$ in $\mathscr{C}_{1}(\mathscr{H})$, the coset $[L]_{Q_{\mu}}$ contains the coset $[L]_{Q_{T}}$, we have $\left\|[L]_{Q_{\mu}}\right\| \leq\left\|[L]_{Q_{T}}\right\|$. Thus $\|u\|,\|v\| \leq(1+\varepsilon)\|[L]\|_{Q_{T}}^{1 / 2}$, and the proof is complete.

The following is our main theorem regarding weak density.

Theorem 2.8. Suppose $\mathcal{M}$ is a weak* closed linear manifold in $\mathscr{L}(\mathscr{H})$ such that every $A$ in $\mathcal{M}$ satisfies $\|A\|=\|A\|_{e}$. Then the set $\left\{[x \otimes y]_{Q_{M}}:\|x\|,\|y\| \leq 1\right\}$ is weakly dense in the unit ball of $Q_{\mu}$.

Proof. Let $\left[L_{0}\right] \in Q_{\mu}$ and satisfy $\left\|\left[L_{0}\right]\right\| \leq 1$. Let $\delta$ be an arbitrary positive number, and let $A_{1}, \ldots, A_{n}$ be an arbitrary finite family from $\mathcal{M}$. Then a typical basic weak neighborhood centered at $\left[L_{0}\right]$ is

$$
\mathcal{N}=\left\{[L] \in Q_{\mu}:\left|\left\langle A_{i},[L]-\left[L_{0}\right]\right\rangle\right|<\delta, i=1, \ldots, n\right\} .
$$

Choose $\varepsilon>0$ so small that

$$
\left|\left\langle A_{i},\left[L_{0}\right]\right\rangle-\left\langle A_{i},\left[L_{0}\right]\right\rangle(1+\varepsilon)^{-2}\right|<\delta, \quad i=1, \ldots, n .
$$

According to Proposition 2.6, there exist vectors $u$ and $v$ in $\mathscr{H}$ such that $\|u\|$, $\|v\| \leq 1+\varepsilon$ and

$$
\left\langle A_{i} u, v\right\rangle=\operatorname{tr}\left(A_{i} L_{0}\right), \quad i=1, \ldots, n .
$$

We define $u^{\prime}=u /(1+\varepsilon), v^{\prime}=v /(1+\varepsilon)$, and compute:

$$
\begin{aligned}
\mid\left\langle A_{i},\left[u^{\prime} \otimes v^{\prime}\right]_{Q_{\mathcal{M}}}\right\rangle-\left\langle A_{i},\right. & {\left.\left[L_{0}\right]_{Q_{\mathcal{M}}}\right\rangle \mid } \\
& =\left|(1+\varepsilon)^{-2}\left(A_{i} u, v\right)-\left\langle A_{i},\left[L_{0}\right]\right\rangle\right| \\
& =\left|(1+\varepsilon)^{-2} \operatorname{tr}\left(A_{i} L_{0}\right)-\left\langle A_{i},\left[L_{0}\right]\right\rangle\right| \\
& =\left|(1+\varepsilon)^{-2}\left\langle A_{i},\left[L_{0}\right]\right\rangle-\left\langle A_{i},\left[L_{0}\right]\right\rangle\right|<\delta, \quad i=1,2, \ldots, n,
\end{aligned}
$$

so $\left[u^{\prime} \otimes v^{\prime}\right] \in \mathcal{N}$, and the proof is complete.

The following immediate consequence of Proposition 2.3 and Theorem 2.8 should be compared with Theorem 2.1, and bears on Problem 2.2.

Theorem 2.9. If $T \in \mathbf{A}$ then the set

$$
\left\{[x \otimes y] \in Q_{T}:\|x\|,\|y\| \leq 1\right\}
$$

is weakly dense in the unit ball of $Q_{T}$.

It follows from the foregoing that to show that every $C_{00}$-contraction $T$ in $\mathscr{L}(\mathscr{H})$ 
satisfying $\sigma(T) \supset \mathbf{T}$ has a nontrivial invariant subspace, it suffices to show that if $T \in \mathbf{A} \cap C_{00}$, then the set $\left\{\phi_{T}([x \otimes y]):\|x\|,\|y\| \leq 1\right\}$, which is weakly dense in the unit ball of $L_{1}(\mathrm{~T}) / H_{0}^{1}(\mathrm{~T})$ by Theorem 2.9 , is also sequentially weakly dense there. Thus this invariant-subspace problem has become, in a certain sense, a function theoretic problem.

\section{REFERENCES}

1. J. Agler, An invariant subspace theorem, J. Funct. Anal. 38 (1980), 315-323.

2. C. Apostol, Ultraweakly closed operator algebras, J. Operator Theory 2 (1979), 49-61.

3. C. ApOSTOL, Invariant subspaces for subquasiscalar operators, J. Operator Theory 3 (1980), 159-164.

4. C. Apostol, A functional calculus and invariant subspaces, J. Operator Theory 4 (1980), 159190.

5. C. ApOSTOL, Invariant subspaces for bilateral weighted shifts, Integral Equations Operator Theory, (to appear).

6. C. Apostol, H. Bercovici, C. Folas \& C. PeArcy, Invariant subspaces, dilation theory, and the structure of the predual of a dual algebra. I, J. Funct. Anal., (to appear).

7. C. Apostol \& B. ChevreAu, On M-spectral sets and rationally invariant subspaces, J. Operator Theory 7 (1982), 247-266.

8. H. BERCOVICI, On dominating sequences in the unit disc, Math. Z., 188 (1984), 33-43.

9. H. BERCOVICI, A reflexivity theorem for weakly closed subspaces of operators, Trans. Amer. Math. Soc., 288 (1985), 139-146.

10. H. Bercovici, B. Chevreau, C. Foias, \& C. Pearcy, Dilation theory and systems of simultaneous equations in the predual of an operator algebra. II, Math. Z. 187 (1984), 97-103.

11. H. Bercovici, C. Foias, J. LANGsam \& C. PeARCy, (BCP)-operators are reflexive, Michigan Math. J. 29 (1982), 371-379.

12. H. BERCOVICI, C. FoIAS \& C. PEARCY, Dilation theory and systems of simultaneous equations in the predual of an operator algebra. I., Michigan Math. J. 30 (1983), 335-354.

13. H. BERCOVICI, C. FOIAS \& C. PEARCY, Factoring trace-class operator-valued functions with applications to the class $\mathbf{A}_{\aleph_{0}}$, J. Operator Theory, (to appear).

14. H. Bercovici, C. Foias, C. PeArCy \& B. Sz.-NAGY, Functional models and generalized spectral dominance, Acta Sci. Math. (Szeged) 43 (1981), 243-254.

15. H. Bercovici, C. FoIAS, C. PeARCy \& B. Sz.-NAGY, Factoring compact operator-valued functions, Acta Sci. Math. (Szeged), (to appear).

16. A. Brown \& C. PEARCY, Introduction to Operator Theory I. Elements of Functional Analysis, Springer, New York, 1977.

17. L. BRown, A. SHIELDS \& K. ZELlER, On absolutely convergent exponential sums, Trans. Amer. Math. Soc. 96 (1960), 162-183.

18. S. BRown, Some invariant subspaces for subnormal operators, Integral Equations Operator Theory 1 (1978), 310-333.

19. S. Brown, B. Chevreau \& C. PeARCY, Contractions with rich spectrum have invariant subspaces, J. Operator Theory 1 (1979), 123-136.

20. B. Chevreau, C. Pearcy \& A. ShIElds, Finitely connected domains $G$, representations of $H^{\infty}(G)$, and invariant subspaces, J. Operator Theory 6 (1981), 375-405.

21. J. DiXMIER, Les Algèbres d'opérateurs dans l'espace Hilbertien (Algèbres de von Neumann), Gauthier-Villars, Paris, 1957.

22. R. Douglas, Banach Algebra Techniques in Operator Theory, Academic Press, New York, 1972.

23. G. EXNER, Systems of equations in the predual of an operator algebra, Ph.D. Thesis, University of Michigan, 1983. 
24. C. Folas, C. PEARCY \& B. Sz.-NAGY, The functional model of a contraction and the space $L^{1}$, Acta Sci. Math. (Szeged) 42 (1980), 201-204.

25. C. Foias, C. Pearcy \& B. Sz.-Nagy, Contractions with spectral radius one and invariant subspaces, Acta Sci. Math. (Szeged), 43 (1981), 273-280.

26. C. FOIAS \& C. PEARCY, (BCP)-operators and enrichment of invariant subspace lattices, J. Operator Theory 9 (1983), 187-202.

27. D. HAdwin \& E. NoRdgren, Subalgebras of reflexive algebras, J. Operator Theory 7 (1982), 3-23.

28. P. Halmos, A Hilbert Space Problem Book, Van Nostrand, Princeton, 1967.

29. D. Herrero, Non-reducing invariant subspaces of normal operators, circulated preprint, 1978.

30. J. LANGSAM, Some results on (BCP)-operators, Ph.D. Thesis, University of Michigan, 1982.

31. R. Olin \& J. Thomson, Algebras of subnormal operators, J. Funct. Anal. 37 (1980), 271-301.

32. R. Olin \& J. Thомsоn, Cellular-indecomposable subnormal operators, Integral Equations Operator Theory 7 (1984), 392-430.

33. C. PEARCY, Some recent results in operator theory, CBMS-MSF Lecture Notes, No. 36, A.M.S., Providence, 1978.

34. G. RoBEL, On the structure of (BCP)-operators and related algebras. I, J. Operator Theory 12 (1984), 23-45.

35. A. SHIELDS, Weighted shift operators and analytic function theory, in "Topics in Operator Theory," (pp. 49-128), Amer. Math. Soc., Providence, 1974.

36. J. StAmPfLI, An extension of Scott Brown's invariant subspace theorem: $K$-spectral sets, J. Operator Theory 3 (1980), 3-22.

37. B. Sz.-NAGY \& C. FoIAS, Harmonic Analysis of Operators on Hilbert Space, North-Holland, Amsterdam, 1970.

38. B. Sz.-NAGY \& C. FoIAS, The function model of a contraction and the space $L^{1} / H_{0}^{1}$, Acta Sci. Math. (Szeged), 41 (1979), 403-410.

39. D. WeSTWOOD, On the structure of the predual of various operator algebras, Ph.D. Thesis, University of Michigan, 1984.

Apostol: Arizona State University-Tempe, AZ 85287

Bercovici: Mathematical Sciences Research Institute-Berkeley, CA 94720

FOIAS: INDIANA UNIVERSITY-BLOOMINGTON, IN 47405

Pearcy: University of Michigan-AnN Arbor, MI 48109

Received November 26, 1984 